Conclusion* Although TEA is not included in ERAS protocols in gynaecological oncology, in experienced hands, it would be a beneficial tool related to decreased need of opioid use and nausea rates with no impact to hospital stay and PO complications, aiming to improve the perioperative quality of patient's care.

\section{LYMPH NODE STATUS AS A PREDICTOR OF VENOUS THROMBOEMBOLIC RISK POSTOPERATIVELY IN GYNAE- ONCOLOGY}

1,2E Ibrahim* ${ }^{*}{ }^{2,3} \mathrm{~L}$ Norris, ${ }^{4} \mathrm{~F}$ Abusaadeh, ${ }^{2,3,5} \mathrm{~S}$ O'toole. ${ }^{1}$ Trinity Centre for Health Sciences, Obstetrics and Gynaecology, Dublin, Ireland; 'Trinity St. James's Cancer Institute, St. James Hospital, Gynaecology, Ireland; ${ }^{3}$ Trinity College Dublin, Obstetrics and Gynaecology, Dublin, Ireland; ${ }^{4}$ St James's Hospital, Gyneoncology , Dublin, Ireland; ${ }^{5}$ Trinity College Dublin, Histopathology, Dublin 8, Ireland

\subsection{6/ijgc-2021-ESG0.306}

Introduction/Background* Gynaecological cancer surgery carries a high risk of venous thromboembolism (VTE). In the absence of thromboprophylaxis, $34.5 \%$ of women with gynaecological cancer develop VTE post operatively compared to $2 \%$ in benign gynaecological surgery patients. Lymph node dissection (LND), an integral part of any gynaecological procedure, carries therapeutic benefit in some cancers but also increases the complications of cancer surgery. An association of LND with VTE has been suggested.

The aim of this study is to investigate the role of LND and lymph node (LN) metastasis on the incidence of VTE following both open and laparoscopic surgery for gynaecological cancer.

Methodology This is a retrospective cohort study analysing data from 1084 patients who underwent gynaecological cancer surgery between 2006-2019 in St James Hospital, Dublin, Ireland (Tertiary referal centre). 1018 patients with complete follow up were included in the study.

Patients with previous VTE, history of significant haemorrhage outside of a surgical setting within the last 5 years, familial bleeding diathesis and patients receiving anticoagulant therapy were excluded. Univariate analysis was used to determine the effects of LND and LN metastasis on the rate of VTE 90 days post surgery.

Result(s)* Forty three patients developed VTE in 90 days post-surgery (4.3\%). VTE rate was significantly higher following open surgery $(5.4 \%)$ compared with laparoscopic approach $(2.3 \%) \quad(\mathrm{P}<0.02)$. The total number of para aortic $\mathrm{LN}$ retrieved significantly increased the rate of VTE $(\mathrm{P}<0.008)$. VTE risk within 90 days was $14.3 \%$ in patients with $>10$ para-aortic LN removed, $5.9 \%$ in patients $<10$ paraaortic LN retrieved, compared with $4.4 \%$ who had no paraaortic LN removed. Pelvic LN metastatic status significantly influenced VTE risk. 5.2\% of patients $<5 \mathrm{LN}$ positive for metastasis had VTE, which increased 4 fold (20\%) in patients with $>5$ LN positive for metastasis $(\mathrm{P}<0.042)$. Lymphovascular space invasion(LVSI) had no effect on VTE risk postoperatively. Overall survival was reduced in patients who developed $\operatorname{VTE}(\mathrm{P}<0.0001)$.

Conclusion* Gynaecological cancer surgery increases VTE risk. The number of paraaortic LN and pelvic LN metastatic status is associated with increased VTE risk and may be useful in predicting VTE post surgery.

\section{AN OVERVIEW OF COMPLICATIONS IN MAJOR GYNAECOLOGICAL ONCOLOGY SURGERY AT A TERTIARY CENTRE}

S Mabbutt* , A Ismail, S Chattopadhyay, Q Davies. University Hospitals of Leicester, UK

\subsection{6/ijgc-2021-ESG0.307}

Introduction/Background* We aim to assess the complication rates across different operative modalities and surgeons. Provide information on patient co-morbidities and tissue diagnosis. This information is important for patient counselling and to provide evidence for ongoing unit accreditation.

Methodology We identified all major gynaecology oncology cases performed at our tertiary centre in 2019, assigned to the 4 oncology surgeons. Cases were assessed for operation type, diagnosis and co-morbidities. Complications then assessed using Clavien-Dindo classification. Data about complications obtained from EDN and follow up clinic letters. Standard used was the UK Gynaecological Oncology Surgical Outcomes and Complications audit of $25.9 \%$ on inclusion of all patientreported complications.

Result(s)* Our major complication rate (Clavien-Dindo 3-4) was $1.61 \%$. Our overall complication rate (Clavien-Dindo 1-4 was $29.8 \%$. 11 deaths recorded, with only 1 death within 28 days of surgerym unrelated to surgery. Of complications, 1 case of intra-abdominal \& retroperitoneal collection, 2 cases wound dehiscence requiring surgical management, 2 cases of haemorrhage requiring relook laparotomy and 1 case returned to theatre for vaginal wall tear after specimen removal.

Conclusion* Our major complication rate is below the national average. Different surgeons have different specialist interests, this may reflect complication rate and allows super specialisation e.g. in robotic surgery. We reported largely similar rates of rare major complications across surgeons and operation type. This knowledge is helpful when consenting patients for procedures, as it gives real life numbers at a local level.

\section{PERIOPERATIVE CARE IN GYNAECOLOGICAL CANCER SURGERY DURING THE COVID PANDEMIC IN A LOW RESOURCE CENTRE - ROLE OF ENHANCED RECOVERY PROTOCOLS}

${ }^{1} \mathrm{PN}$ Rema*,${ }^{1} \mathrm{~A}$ Nath, ${ }^{1} \mathrm{D}$ Dinesh, ${ }^{2} \mathrm{~S}$ Ranjith J, 'S Sambasivan. ${ }^{1}$ Regional cancer center Thiruvananthapuram, Department of Gynaecological oncology, Thiruvananthapuram, India; ${ }^{2}$ Regional cancer center Thiruvananthapuram, Department of surgical oncology, Thiruvananthapuram, India

\subsection{6/ijgc-2021-ESG0.308}

Introduction/Background* ERAS (Enhanced Recovery after Surgery) is a multimodal perioperative care pathway designed to achieve early recovery after surgical procedures. This study aimed to analyse the feasibility of ERAS in the era of pandemic and to find its effect on the post-operative outcome of patients undergoing surgery for gynaecological cancer during the COVID pandemic

Methodology This observational study was done on patients who underwent gynaecological cancer surgery during COVID pandemic in a tertiary cancer centre in South India. Data was collected including patient demographics, nature of surgery, adherence to each of the components of ERAS programme 